\title{
Predictive Factors for Differentiating Gastrointestinal Stromal Tumors from Leiomyomas Based on Endoscopic Ultrasonography Findings in Patients with Gastric Subepithelial Tumors: A Multicenter Retrospective Study
}

\author{
Sun Moon Kim ', Eun Young Kim², Jin Woong Cho ${ }^{3}$, Seong Woo Jeon ${ }^{4}$, Ji Hyun Kim ${ }^{5}$, Tae Hyeon Kim ${ }^{6}$, Jeong Seop Moon ${ }^{7}$ Jin-Oh \\ $\mathrm{Kim}^{8}$, the Research Group for Endoscopic Ultrasound of the Korean Society of Gastrointestinal Endoscopy \\ ${ }^{1}$ Department of Internal Medicine, Konyang University College of Medicine, Daejeon, ${ }^{2}$ Department of Internal Medicine, Daegu Catholic \\ University School of Medicine, Daegu, ${ }^{3}$ Department of Internal Medicine, Presbyterian Medical Center, Jeonju, ${ }^{4}$ Department of Internal \\ Medicine, Kyungpook National University Chilgok Hospital, Daegu, ${ }^{5}$ Department of Internal Medicine, Inje University College of \\ Medicine, Busan, ${ }^{6}$ Department of Internal Medicine, Wonkwang University College of Medicine and Hospital, Iksan, ${ }^{7}$ Department of \\ Internal Medicine, Inje University College of Medicine, Seoul, ${ }^{8}$ Department of Internal Medicine, Soonchunhyang University College of \\ Medicine, Seoul, Korea
}

Background/Aims: The utility of endoscopic ultrasonography (EUS) for differentiating gastrointestinal stromal tumors (GISTs) and leiomyomas of the stomach is not well known. We aimed to evaluate the ability of EUS for differentiating gastric GISTs and leiomyomas.

Methods: We retrospectively reviewed the medical records of patients with histopathologically proven GISTs ( $n=274)$ and leiomyomas $(n=87)$. In two consensus meetings, the inter-observer variability in the EUS image analysis was reduced. Using logistic regression analyses, we selected predictive factors and constructed a predictive model and nomogram for differentiating GISTs from leiomyomas. A receiver operating characteristic (ROC) curve analysis was performed to measure the discrimination performance in the development and internal validation sets.

Results: Multivariate analysis identified heterogeneity (odds ratio [OR], 9.48), non-cardia (OR, 19.11), and older age (OR, 1.06) as independent predictors of GISTs. The areas under the ROC curve of the predictive model using age, sex, and four EUS factors (homogeneity, location, anechoic spaces, and dimpling or ulcer) were 0.916 (sensitivity, 0.908; specificity, 0.793 ) and 0.904 (sensitivity, 0.908; specificity, 0.782 ) in the development and internal validation sets, respectively.

Conclusions: The predictive model and nomogram using age, sex and homogeneity, tumor location, presence of anechoic spaces, and presence of dimpling or ulcer on EUS may facilitate differentiation between GISTs and leiomyomas. Clin Endosc 2021;54:872-880

Key Words: Endosonography; Gastrointestinal stromal tumors; Leiomyoma; Stomach

\section{INTRODUCTION}

Gastric subepithelial tumors (SETs) are usually found inci- dentally as a firm protruding mass in individuals undergoing esophagogastroduodenoscopy (EGD). The diagnosis of gastric SETs is increasing in Korea because of the widespread use of

\footnotetext{
Received: October 8, 2021 Revised: October 17, 2021

Accepted: October 18, 2021

Correspondence: Eun Young Kim

Department of Internal Medicine, Daegu Catholic University School of Medicine, 33 Duryugongwon-ro 17-gil, Nam-gu, Daegu 42472, Korea

Tel: +82-53-650-4092, Fax: +82-53-621-4487, E-mail: kimey@cu.ac.kr ORCID: https://orcid.org/0000-0003-3965-9964
}

(c) This is an Open Access article distributed under the terms of the Creative Commons Attribution Non-Commercial License (http://creativecommons.org/licenses/by$\mathrm{nc} / 3.0$ ) which permits unrestricted non-commercial use, distribution, and reproduction in any medium, provided the original work is properly cited. 
endoscopy as part of a national screening program for gastric cancer. Its incidence was reportedly $0.76-1.94 \%$ in recent studies. ${ }^{1,2}$ Gastric SETs present as benign, potentially malignant, or malignant lesions. Most SETs have a benign clinical course. Some tumors, including lymphomas, gastrointestinal stromal tumors (GISTs), neuroendocrine tumors, and glomus tumors, are malignant or potentially malignant. ${ }^{3}$

Gastric SETs are extremely difficult to differentiate using conventional endoscopy alone. Endoscopic ultrasonography (EUS) has been used to differentiate gastric SETs and plays a crucial role in confirming the diagnosis in some cases. However, it is particularly difficult to differentiate gastric SETs within the fourth (muscularis propria) echolayer. ${ }^{4}$ Among them, GISTs and leiomyomas are the most common mesenchymal tumors noted in the muscularis propria of the stomach. ${ }^{5,6}$ Distinguishing between GISTs and leiomyomas is essential to guide effective clinical management, as GISTs are potentially malignant, whereas leiomyomas are benign. ${ }^{7}$

Although several studies have attempted to distinguish SETs based on EUS findings, their results were unsatisfactory. ${ }^{8,9}$ Moreover, most were single-center retrospective studies with small sample sizes. Using EUS findings, this multicenter study aimed to elucidate predictive factors and subsequently create a predictive risk score model and nomogram for differentiating gastric GISTs and leiomyomas.

\section{METHODS}

\section{Patients}

We retrospectively reviewed the medical records of patients with histopathologically proven gastric GISTs or leiomyomas originating from the fourth layer who underwent EUS examinations at eight university hospitals (members of the Research Group for Endoscopic Ultrasound of the Korean Society of Gastrointestinal Endoscopy) between January 2005 and December 2015. For the immunohistochemical analysis, GISTs were defined as positive for c-KIT, while leiomyomas were defined as positive for desmin or smooth muscle actin.

We enrolled 361 patients (162 men and 199 women) with a mean age of 57 years (range, 26-87 years), of whom 274 had GISTs and 87 had leiomyomas. The pathological findings were confirmed using tissues obtained surgically (306 patients [84.8\%]), biopsy with endoscopic forceps (37 patients [10.2\%]), EUS-guided fine-needle biopsy (EUS-FNB) (13 patients [3.6\%]), or endoscopic resection (5 patients [1.4\%]), including endoscopic mucosal resection or endoscopic submucosal dissection.

This study was performed in accordance with the ethical guidelines of the Declaration of Helsinki and approved by the Institutional Review Board of Konyang University Hospital (approval no. 2015-07-015).

\section{Patient data}

From the medical records including EGD and EUS images, we obtained data on patient demographics and tumor characteristics, including location (non-cardia, cardia), tumor shape (non-elongated, elongated), tumor growth pattern (endophytic, exophytic, mixed), and the presence of dimpling or ulcer, mucosal erythema, and lobulation. Data pertaining to maximal diameter, echogenicity (hyperechoic, isoechoic, hypoechoic), homogeneity (homogenous, heterogeneous), presence of anechoic spaces, presence of hyperechoic spots, and marginal regularity (regular, irregular) were recorded based on the EUS findings. Echogenicity was determined by comparison with the normal muscularis propria layer. The term "homogenous" was defined as more than $75 \%$ of the total area appearing homogenous.

The interpretation of EUS images involves many inter-observer differences. Therefore, we collected three typical cases from each hospital and selected 20 cases worthy of discussion. Two consensus meetings were held on the EUS findings in these cases. After consensus meetings, the cases from each hospital were interpreted independently by endosonographers according to the criteria determined at the meeting, and the results were collected.

\section{Statistical analysis}

In this study, we evaluated the inter-observer agreement of the five EUS findings using Fleiss's kappa ( $\mathrm{k}$ ) to compare their reliability. A $\mathrm{k}$ value of 1.0 indicates perfect agreement. Values of $\mathrm{K}>0.80$ were considered "excellent," $0.60-0.79$ were considered "good," $0.40-0.59$ were considered "fair," and $<0.40$ were considered "poor."

Categorical variables were analyzed using the $\chi^{2}$ test or Fisher's exact test. Continuous variables were analyzed using Student's $t$-test or the Mann-Whitney $U$-test. Univariate binary logistic regression was used to assess the effectiveness of potential predictive factors in differentiating GISTs from leiomyomas in patients with SETs. A multivariate predictive model was then constructed using a backward stepwise variable elimination procedure including the predictive factors with values of $p<0.1$ in the univariate analysis.

Receiver operating characteristic (ROC) curve analysis was performed to measure the discrimination performance in the development and internal validation sets. The reliability of the constructed model was internally validated using leave-oneout cross-validation. For practical application of the prediction 
model in the clinical field, a nomogram was constructed using the selected predictors.

Statistical significance was set at $p<0.05$. The statistical analyses were performed using $\mathrm{R}$ version 3.3.3 ( $\mathrm{R}$ Foundation for Statistical Computing, Vienna, Austria) and T\&F program ver. 2.8 (YooJin BioSoft Co., Ltd, Goyang, Korea).

\section{RESULTS}

\section{Inter-observer variability of the five EUS features}

Before the first consensus meeting, the average $\mathrm{K}$ value for the inter-observer variability of the five EUS features was 0.178 . Agreement for all EUS findings was poor, with a $\mathrm{k}$ value of $<0.40$. After the first consensus meeting, the average $\mathrm{K}$ value improved to 0.281 , but the agreement was still poor. The agreement for homogeneity was fair $(\mathrm{k}=0.41)$, but that for the other four EUS factors was poor. After the second consensus meeting, the average $\mathrm{K}$ value improved to 0.459 , and the agreement was fair. There was good agreement for hyperechoic spots $(\mathrm{K}=0.724)$, fair agreement for homogeneity and anechoic spaces ( $\mathrm{K}=0.459$ and 0.507 , respectively), and poor agreement for echogenicity and a marginal irregularity $(\mathrm{K}=0.353$ and 0.257 , respectively) (Table 1 ).

\section{Predictive factors differentiating GISTs from leiomyomas}

The clinicopathologic characteristics and EUS findings are presented in Table 2. The maximum tumor sizes of GISTs and leiomyomas were 11.7 and $5.7 \mathrm{~cm}$, respectively. On univariate analysis, GISTs were significantly associated with male sex, older age, non-cardia, dimpling or ulcer on the tumor surface, non-elongated shape, exophytic or mixed tumor growth pattern, absence of lobulation, large tumor size, higher echogenicity, tumor heterogeneity, and presence of anechoic spaces. The presence of hyperechoic spots, mucosal erythema, and irregular tumor borders were not significantly different between
GISTs and leiomyomas.

The multivariate analysis revealed that heterogeneity (odds ratio [OR], 9.48; 95\% confidence interval [CI], 3.30-27.27), non-cardia (OR, 19.11; 95\% CI, 9.36-39.02), and older age (OR, 1.06; 95\% CI, 1.02-1.09) were independent predictive factors for differentiating gastric GISTs and leiomyomas originating from the fourth layer (Table 3; Fig. 1).

\section{Predictive model for differentiating GISTs and leiomyomas based on EUS findings in patients with gastric SETs}

Based on the results of the univariate and multivariate analyses, we selected six predictive factors and developed a predictive model for differentiating GISTs and leiomyomas that originate from the muscularis propria layer as shown below:

$$
\text { Probability to be case sample }=\mathrm{e}^{\beta \mathrm{X}} /\left(1+\mathrm{e}^{\beta \mathrm{X}}\right) \text {, }
$$

where $\beta X=-4.044+(0.056 \times$ age $)-(0.706 \times$ sex $)+(2.25$ $\times$ homogeneity $)+(1.311 \times$ anechoic spaces $)+(2.95 \times$ location $)+(0.867 \times$ ulceration or dimpling $)$. In this predictive model, the values used were " 0 " for female sex, homogeneity, and absence of anechoic spaces, cardia, and absence of dimpling or ulcer, respectively. The values were "1" for male sex, heterogeneity, presence of anechoic spaces, non-cardia, and presence of dimpling or ulcer.

As this equation is too complex for use in the clinical field, a nomogram was constructed to estimate the predicted probability of the GISTs (Fig. 1). For each predictive factor in the nomogram, the point was read out by drawing a line straight upward from each predictor to the point axis. In the nomogram, the values were "0" for age $<60$ years, female sex, homogeneity, absence of anechoic spaces, cardia site, and absence

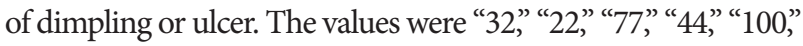
and " 18 " for age $60 \geq$ years, male sex, heterogeneity, presence of anechoic spaces, non-cardia site, and presence of dimpling or ulcer, respectively. The total points of these variables can be

Table 1. Kappa Values for Inter-Observer Variability of the Five Endoscopic Ultrasonography Features

\begin{tabular}{lccc}
\hline & Before consensus meeting & After first meeting & After second meeting \\
\hline Echogenicity & 0.025 & 0.158 & 0.353 \\
Homogeneity & 0.317 & 0.406 & 0.459 \\
Presence of hyperechoic spots & 0.106 & 0.372 & 0.724 \\
Presence of anechoic spaces & 0.384 & 0.257 & 0.507 \\
Marginal irregularity & 0.059 & 0.213 & 0.252 \\
Average kappa value & 0.178 & 0.281 & 0.459 \\
\hline
\end{tabular}


Table 2. Baseline Characteristics and Endoscopic Ultrasonography Findings of the Patients with Gastrointestinal Stromal Tumors and Leiomyomas of the Stomach

\begin{tabular}{|c|c|c|c|}
\hline Variable & GIST $(n=274)$ & Leiomyoma $(n=87)$ & $P$ value \\
\hline Sex & & & 0.047 \\
\hline Male & $131(47.8 \%)$ & $31(35.6 \%)$ & \\
\hline Female & $143(52.2 \%)$ & $56(64.4 \%)$ & \\
\hline Age (years, mean $\pm S D$ ) & $60.3 \pm 11.8$ & $51.3 \pm 12.2$ & $<0.001$ \\
\hline Location & & & $<0.001$ \\
\hline Cardia & $34(12.4 \%)$ & $63(72.4 \%)$ & \\
\hline Antrum/body/fundus & $240(87.6 \%)$ & $24(27.6 \%)$ & \\
\hline Dimpling or ulcer & & & 0.003 \\
\hline Absent & $222(81.0 \%)$ & $82(94.3 \%)$ & \\
\hline Present & $52(19.0 \%)$ & $5(5.7 \%)$ & \\
\hline Tumor shape & & & 0.008 \\
\hline Non-elongated & $215(78.5 \%)$ & $56(64.4 \%)$ & \\
\hline Elongated & $59(21.5 \%)$ & $31(35.6 \%)$ & \\
\hline Mucosal erythema & & & 0.167 \\
\hline Absent & $218(79.6 \%)$ & $75(86.2 \%)$ & \\
\hline Present & $56(20.4 \%)$ & $12(13.8 \%)$ & \\
\hline Tumor growth & & & $<0.001$ \\
\hline Endophytic & $127(46.4 \%)$ & $65(74.7 \%)$ & \\
\hline Exophytic & $103(37.6 \%)$ & $16(18.4 \%)$ & \\
\hline Mixed & $44(16.1 \%)$ & $6(6.9 \%)$ & \\
\hline Lobulation & & & 0.005 \\
\hline Absent & $225(82.1 \%)$ & $59(67.8 \%)$ & \\
\hline Present & $49(17.9 \%)$ & $28(32.2 \%)$ & \\
\hline Size $(\mathrm{cm})$ & & & $<0.001$ \\
\hline Mean \pm SD & $2.91 \pm 1.7$ & $2.04 \pm 1.01$ & 0.001 \\
\hline$<2.0$ & $77(28.1 \%)$ & $44(50.6 \%)$ & \\
\hline $2-3.5$ & $129(47.1 \%)$ & $37(42.5 \%)$ & \\
\hline$>3.5$ & $68(24.8 \%)$ & $6(6.9 \%)$ & \\
\hline Echogenicity & & & 0.002 \\
\hline Hypoechoic & $104(38 \%)$ & $43(49.4 \%)$ & \\
\hline Isoechoic & $119(43.4 \%)$ & $41(47.1 \%)$ & \\
\hline Hyperechoic & $51(18.6 \%)$ & $3(3.5 \%)$ & \\
\hline Homogeneity & & & $<0.001$ \\
\hline Homogenous & $159(58.0 \%)$ & $81(93.1 \%)$ & \\
\hline Heterogenous & $115(42.0 \%)$ & $6(6.9 \%)$ & \\
\hline Hyperechoic spots & & & 0.28 \\
\hline Absent & $208(75.9 \%)$ & $61(70.1 \%)$ & \\
\hline Present & $66(24.1 \%)$ & $26(29.9 \%)$ & \\
\hline Anechoic spaces & & & $<0.001$ \\
\hline Absent & $208(75.9 \%)$ & $84(96.6 \%)$ & \\
\hline Present & $66(24.1 \%)$ & $3(3.4 \%)$ & \\
\hline Marginal irregularity & & & 0.127 \\
\hline Regular & 247 (90.1\%) & $83(95.4 \%)$ & \\
\hline Irregular & $27(9.9 \%)$ & $4(4.6 \%)$ & \\
\hline
\end{tabular}

GIST, gastrointestinal stromal tumor; SD, standard deviation. 
Table 3. Univariate and Multivariate Analysis of Endoscopic Ultrasonography Features

\begin{tabular}{|c|c|c|c|c|c|}
\hline \multirow{2}{*}{ Variable } & & \multicolumn{2}{|c|}{ Univariate analysis } & \multicolumn{2}{|c|}{ Multivariate analysis } \\
\hline & & OR (95\% CI) & $P$ value & OR (95\% CI) & $P$ value \\
\hline Age (years) & $\geq 60$ vs. $<60$ & $1.06(1.04-1.09)$ & $<0.001$ & $1.06(1.02-1.09)$ & $<0.001$ \\
\hline Sex & Female vs. male & $0.60(0.37-0.99)$ & 0.048 & $0.49(0.24-1.01)$ & 0.054 \\
\hline Echogenicity & $\begin{array}{l}\text { Isoechoic vs. hypoechoic } \\
\text { Hyperechoic vs. hypoechoic }\end{array}$ & $\begin{array}{c}1.2(0.73-1.98) \\
7.03(2.08-23.75\end{array}$ & $\begin{array}{l}0.007 \\
0.477 \\
0.002\end{array}$ & & \\
\hline Homogeneity & Heterogenous vs. homogenous & $9.83(4.14-23.30)$ & $<0.001$ & $9.48(3.30-27.27)$ & $<0.001$ \\
\hline Hyperechoic spots & Present vs. absent & $0.74(0.44-1.27)$ & 0.281 & & \\
\hline Anechoic spaces & Present vs. absent & $8.89(2.72-29.04)$ & $<0.001$ & $3.71(0.93-14.81)$ & 0.063 \\
\hline Marginal irregularity & Irregular vs. regular & $2.27(0.77-6.67)$ & 0.137 & & \\
\hline Location & Non-cardia vs. cardia & $18.53(10.26-33.48)$ & $<0.001$ & $19.11(9.36-39.02)$ & $<0.001$ \\
\hline Dimpling or ulcer & Present vs. absent & $3.84(1.48-9.95)$ & 0.006 & $2.38(0.68-8.33)$ & 0.175 \\
\hline Tumor shape & Elongated vs. non-elongated & $0.496(0.29-0.83)$ & 0.009 & & \\
\hline Mucosal erythema & Present vs. absent & $1.61(0.81-2-3.16)$ & 0.17 & & \\
\hline
\end{tabular}

$\mathrm{CI}$, confidence interval; OR, odds ratio.

Points

Age

Sex

Homogeneity

Anechoic spaces

Location

Ulcer

Total points

Probability of risk

$\begin{array}{lllllllllll}0 & 10 & 20 & 30 & 40 & 50 & 60 & 70 & 80 & 90 & 100\end{array}$

$>=60$

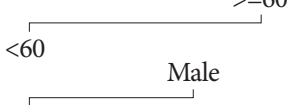

$$
\text { Female }
$$

Heterogenous

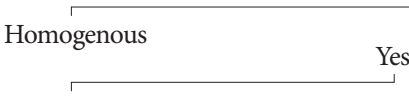

No

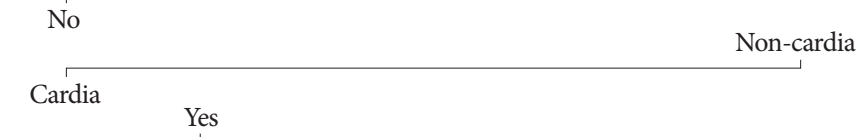

No

$\begin{array}{rrrrrrr}1 & 100 & 150 & 200 & 250 & 300\end{array}$

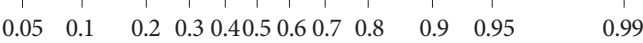

Fig. 1. Nomogram to estimate the predicted probability for differentiating gastrointestinal stromal tumors and leiomyomas based on endoscopy and endoscopic ultrasonography findings in patients with gastric subepithelial lesions that originated from the muscularis propria layer.

translated into the probability of risk.

For example, in the representative GIST patient shown in Fig. 2, the sum of the values is 209 (age $60 \geq$ years [32] + heterogeneity [77] + non-cardia site [100]), which corresponds to a risk probability of 0.994 . This result is strongly predictable for GISTs. In the leiomyoma case shown in Fig. 3, the sum of the values is 22 (male sex [22]), which corresponds to a risk probability of 0.204 . This result indicates a high probability of leiomyoma.

The area under the ROC curve of the predictive model was 0.916 (95\% CI, 0.881-0.950) (Fig. 4). The optimal cutoff was 0.708 , which was computed at the point maximizing Youden's J statistic. In the development set, the sensitivity, specificity, accuracy, positive predictive value, and negative predictive value 

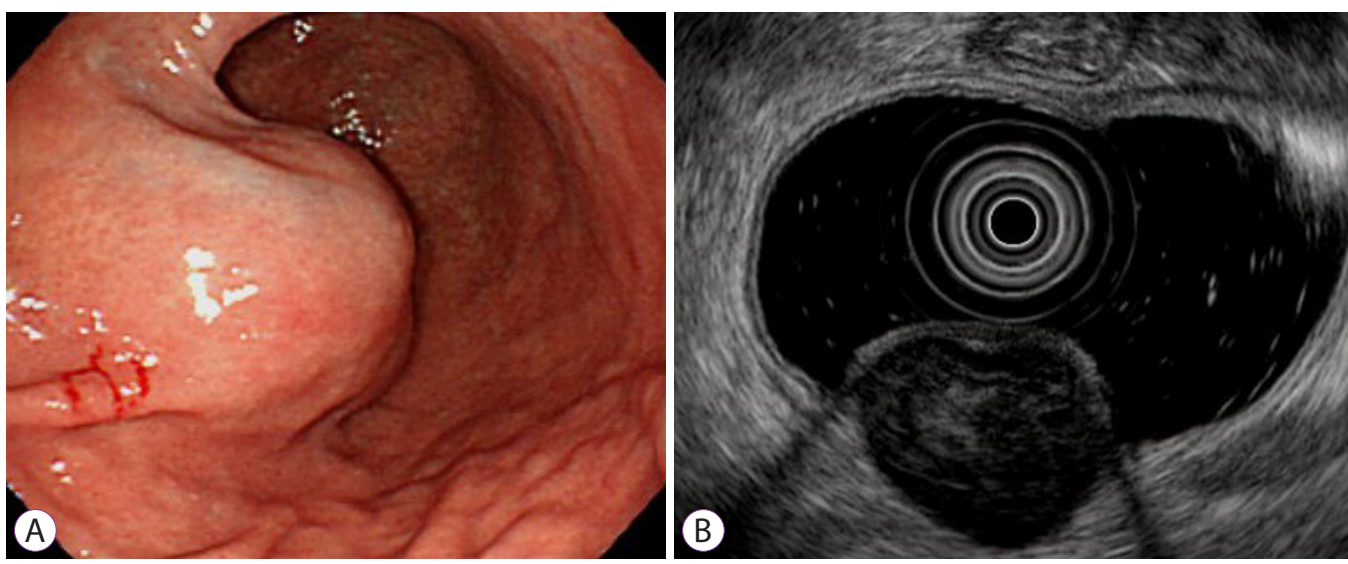

Fig. 2. A gastrointestinal stromal tumor (GIST) in a 65-year-old woman. (A) Endoscopic image showing a round subepithelial tumor in the lower body of the stomach. (B) Endoscopic ultrasonogram demonstrating a heterogenous isoechoic mass originating from the fourth layer. It is $3.5 \times 3.0 \mathrm{~cm}$ in size and shows multiple internal hyperechoic spots and distinct margin. According to the nomogram, the sum of values is 209 , corresponding to a risk probability of 0.994 . This result strongly predicts the GIST.
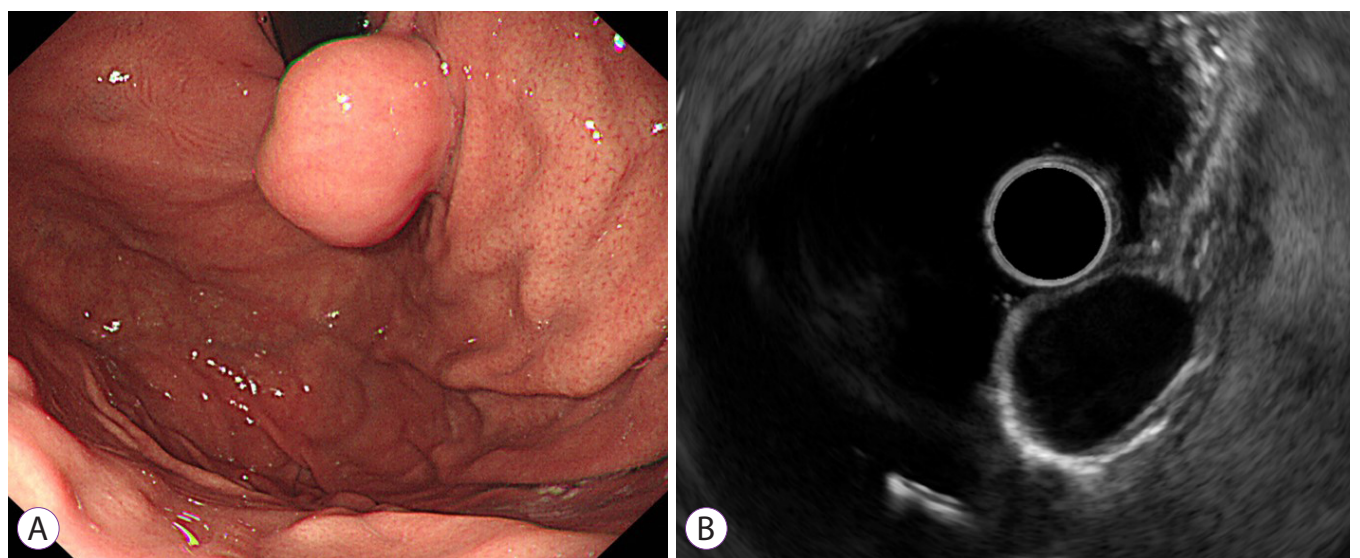

Fig. 3. A leiomyoma in a 40-year-old man. (A) Endoscope image showing an elongated subepithelial tumor without a mucosal ulcer in the gastric cardia. (B) Endoscopic ultrasonogram revealing a homogenous hypoechoic mass without anechoic spaces or hyperechoic spots arising from the fourth layer measuring $2.1 \times 1.5 \mathrm{~cm}$ in size. According to the nomogram, the sum of values is 22 , corresponding to a risk probability of 0.204 . This result indicates a high probability of a leiomyoma.
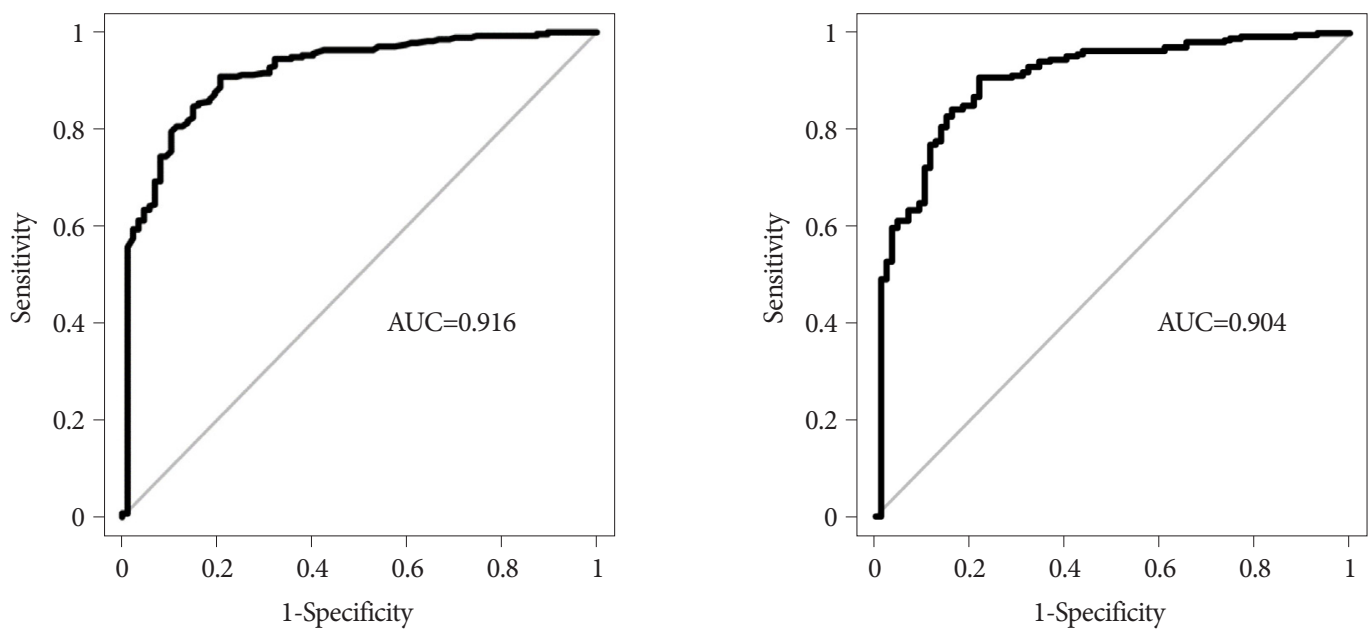

Fig. 4. Receiver operating characteristic curve of the discrimination accuracy of the prediction model integrating two endoscopic findings (location and presence of dimpling or ulcer) and two endosonographic findings (homogeneity and anechoic spaces) adjusted for age and sex for differentiating gastrointestinal stromal tumors from leiomyomas. (A) Development set. (B) Internal validation set. AUC, area under the curve. 
were $90.8 \%, 79.3 \%, 88.1 \%, 93.2 \%$, and $73.4 \%$, respectively.

The internal validation of this predictive model was assessed using a leave-one-out cross-validation procedure. The area under the ROC curve of the predictive model was 0.904 (95\% CI, 0.867-0.941), and the optimal cutoff value was 0.712 . The ROC curves were similar between the developed model and the internal validation set, and the model showed good discriminative power in both datasets. In the internal validation set, the sensitivity, specificity, accuracy, positive predictive value, and negative predictive value, with a cutoff point of 0.712 , were $90.8 \%, 78.2 \%, 87.8 \%, 92.9 \%$, and $73.1 \%$, respectively.

\section{Predictive factors and model for differentiating GISTs from leiomyomas using tumors of different sizes}

\section{Tumors smaller than $2 \mathrm{~cm}$}

We found 121 gastric SETs $<2 \mathrm{~cm}$, including 77 GISTs and 44 leiomyomas. After univariate and multivariate analyses, age, sex, homogeneity, and tumor location were independent predictive factors for differentiating gastric GISTs and leiomyomas based on the EUS finding. The area under the ROC curve of the predictive model using age, sex, homogeneity, and tumor location was similar between the two datasets. In the development and internal validation sets, the area under the curve was 0.841 (95\% CI, 0.762-0.919) and 0.815 (95\% CI, 0.731-0.899), respectively.

\section{Tumors with 2-3.5 $\mathrm{cm}$ in size}

A total of 129 GISTs and 37 leiomyomas were $2.0-3.5 \mathrm{~cm}$ in size. After the univariate and multivariate analyses, age and homogeneity were independent predictive factors for distinguishing gastric GISTs and leiomyomas. The areas under the ROC curve of this predictive model with age, homogeneity, and sex in gastric SETs were 0.812 (95\% CI, 0.733-0.892) and 0.787 (95\% CI, 0.7-0.873) in the development and validation sets, respectively.

\section{Tumors larger than $3.5 \mathrm{~cm}$}

There were 68 GISTs and 6 leiomyomas larger than $3.5 \mathrm{~cm}$. After the multivariate analysis, age, tumor shape, and hyperechoic spots were independent predictive factors for differentiating gastric GISTs and leiomyomas. The area under the ROC curve of the predictive model using age, tumor shape, hyperechoic spots, and sex was 0.968 (95\% CI, 0.922-1.000) and 0.654 (95\% CI, 0.248-1.000) in the development and internal validation sets, respectively. This model showed good discriminative power in the development set but not in the validation set.

\section{DISCUSSION}

In this multicenter retrospective study, we aimed to investigate the predictive factors and develop a predictive risk score model and nomogram for differentiating gastric GISTs and leiomyomas based on EUS findings. The multivariate analysis identified heterogeneity, non-cardia, and older age as independent predictors of GISTs. The areas under the ROC curve of the predictive model using age, sex, and four EUS factors (heterogeneity, location, anechoic spaces, and dimpling or ulcer) were 0.916 (sensitivity, 0.908; specificity, 0.793) and 0.904 (sensitivity, 0.908; specificity, 0.782) in the development and internal validation sets, respectively.

EUS is the most valuable diagnostic tool for evaluating the layer of origin, size, internal echogenicity, margin, and echotexture of SETs. ${ }^{10}$ However, although several studies have aimed to diagnose SETs using EUS, the diagnostic accuracy was relatively low for the differential diagnosis of such tumors, particularly for those that originate from the muscularis propria layer. ${ }^{8,9,11}$ In addition, since the interpretation of EUS findings of gastric SETs has significant inter-observer variability, most studies of SETs have been single-center studies with small sample sizes. ${ }^{12}$ Thus, we here planned a large sample size. Additionally, two consensus meetings were held to overcome inter-observer variability in the analysis of the EUS images, which is considered the largest problem with this approach. The first interpretation was based on existing personal criteria. The $\mathrm{k}$ values for inter-observer variability were very low $(\mathrm{k}<0.40)$, and the agreement of the five EUS findings was poor. After the first meeting, the average $\mathrm{k}$ value improved to 0.28 , but the agreement of four EUS findings, except homogeneity, remained poor. Consequently, we conducted an additional consensus meeting. The average $\mathrm{k}$ value of the third interpretation improved to 0.459 and the agreement was fair. The agreement for hyperechoic spots was good, whereas that for homogeneity and anechoic spaces was fair. Unfortunately, the agreement on the echogenicity and the marginal irregularity remained poor after the second meeting. To our knowledge, no report to date has evaluated the inter-observer agreement of EUS findings of gastric SETs. Despite these two meetings, the agreement improved only somewhat and not satisfactorily. Thus, we confirmed that the interpretation of EUS findings of gastric SETs has significant inter-observer variability.

In this study, GISTs were more common in the gastric antrum, body, and fundus. In contrast, leiomyomas were more common in the cardia. These results were similar to those reported in previous studies. ${ }^{13,14}$ Most leiomyomas do not require surgery. In recent years, most SET operations have been performed using laparoscopic wedge resection. The cardia 
area is known to be a very difficult operation site because of the technical complexities of the procedure and complications such as gastroesophageal reflux or late stenosis. ${ }^{14}$ When considering surgery in SETs in the cardia, efforts for preoperative pathologic diagnosis, such as EUS-FNB, unroofing technique, and single-incision needle-knife biopsy, are needed to avoid unnecessary surgery.

In a previous study, homogeneity, echogenicity, the presence of hyperechogenic spots and anechoic spaces, older age, and presence of a marginal halo were reported EUS findings for differentiating GISTs from leiomyomas. ${ }^{8,15,16}$ In the present study, older age, tumor inhomogeneity, and non-cardia site were independent predictive factors for GISTs on multivariate analysis. In contrast, echogenicity, the presence of hyperechogenic spots and anechoic spaces, tumor shape, mucosal erythema, and marginal regularity were not helpful for predicting GISTs. In fact, among the important EUS findings, homogeneity was the only predictive factor.

The current study is the first to analyze the characteristics of tumor by size groups including tumors with $0.6-11.7 \mathrm{~cm}$ in size. In SETs smaller than $2 \mathrm{~cm}$, age, sex, homogeneity, and tumor location were significant independent predictive factors for differentiating GISTs and leiomyomas. In tumors with $2-3.5 \mathrm{~cm}$ in size, age and homogeneity were significant, while in tumors larger than $3.5 \mathrm{~cm}$, age, tumor shape, and hyperechoic spots were significant.

In the development set, the area under the ROC curve in gastric SETs smaller than $2.0 \mathrm{~cm}, 2.0-3.5 \mathrm{~cm}$, and larger than $3.5 \mathrm{~cm}$ were $0.841,0.812$, and 0.968 , respectively. We were mostly interested in gastric SETs with $2.0-3.5 \mathrm{~cm}$ in size, which decided whether to undergo surgery according to histopathology of SETs, but the discriminative ability of EUS was lowest for tumors of this size range.

Moreover, in the internal validation set, SETs smaller than 2.0 $\mathrm{cm}$ and with $2.0-3.5 \mathrm{~cm}$ in size showed areas under the curve similar to those of the development set. However, SETs larger than $3.5 \mathrm{~cm}$ had a lower area under the curve, which was most likely due to the small number of leiomyomas larger than 3.5 $\mathrm{cm}$.

In the present study, we developed a predictive risk score model for differentiating gastric GISTs and leiomyomas that originate from the muscularis propria layer based on EUS findings. If the SETs were diagnosed as GISTs with a cutoff level of 0.708 , the sensitivity, specificity, and accuracy of our model were $0.908,0.793$, and 0.881 , respectively. This level of accuracy was noteworthy. Therefore, this model might be a complementary method to various other methods currently applied for discrimination of GISTs.

However, this study has some limitations. First, it was ret-

rospective and used endosonographic images; therefore, there might have been selection bias. Second, several endoscopists independently interpreted the EUS findings of the gastric SETs. Although two consensus meetings were held to reduce inter-observer variability, agreement ended up just "fair". Finally, this study included small SETs.

Despite these limitations, the present study is important because it involved a multicenter trial that used two consensus meetings to reduce inter-observer variability and included a relatively large number of patients with leiomyoma. In addition, no previous study performed an analysis by tumor size.

In conclusion, EUS features such as heterogeneity, non-cardia site, and older age were significant independent factors for differentiating gastric GISTs from leiomyomas. Additionally, the predictive model and nomogram developed in the present study, which uses age, sex, homogeneity, the presence of anechoic spaces, tumor location, and the presence of dimpling or ulcer, may be helpful for differentiating GISTs from leiomyomas.

\title{
Conflicts of Interest
}

Eun Young Kim is currently serving as a deputy editor in Clinical Endoscopy; however, she had not involved in the peer reviewer selection, evaluation, or decision process of this article. The authors have no potential conflicts of interest.

\section{Funding}

This study was supported by the Gastrointestinal Endoscopy Research Foundation of Korea, an Olympus Grant 2015 and the Korean Society of Gastrointestinal Endoscopy (Seoul, Korea).

\author{
Author Contributions \\ Conceptualization: Sun Moon Kim, Eun Young Kim \\ Data curation: SMK, EYK, Jin Woong Cho, Seong Woo Jeon, Ji Hyun \\ Kim, Tae Hyeon Kim, Jeong Seop Moon, Jin-Oh Kim \\ Funding acquisition: SMK \\ Formal analysis: SMK \\ Investigation: SMK, EYK, JWC, SWJ, JHK, THK, JSM, JOK \\ Methodology: SMK, EYK \\ Project administration: SMK, EYK, JWC, SWJ, JHK, THK, JSM, JOK \\ Validation: SMK, EYK \\ Writing - original draft: SMK \\ Writing - review and editing: SMK, EYK
}

\section{ORCID}

Sun Moon Kim: Eun Young Kim: Jin Woong Cho: Seong Woo Jeon: Ji Hyun Kim: Tae Hyeon Kim: Jeong Seop Moon: Jin-Oh Kim: https://orcid.org/0000-0002-0436-3381 https://orcid.org/0000-0003-3965-9964 https://orcid.org/0000-0002-0296-8045 https://orcid.org/0000-0002-9539-9389 https://orcid.org/0000-0003-0861-2792 https://orcid.org/0000-0002-9723-2136 https://orcid.org/0000-0002-5909-8159 https://orcid.org/0000-0001-7036-3155 


\section{REFERENCES}

1. Lee JH, Lee HL, Ahn YW, et al. Prevalence of gastric subepithelial tumors in Korea: a single center experience. Korean J Gastroenterol 2015;66:274-276.

2. Lim YJ, Son HJ, Lee JS, et al. Clinical course of subepithelial lesions detected on upper gastrointestinal endoscopy. World J Gastroenterol 2010;16:439-444.

3. Hwang JH, Rulyak SD, Kimmey MB, American Gastroenterological Association Institute. American Gastroenterological Association Institute technical review on the management of gastric subepithelial masses. Gastroenterology 2006;130:2217-2228.

4. Cho JW, Korean ESD Study Group. Current guidelines in the management of upper gastrointestinal subepithelial tumors. Clin Endosc 2016;49:235-240.

5. Menon L, Buscaglia JM. Endoscopic approach to subepithelial lesions. Therap Adv Gastroenterol 2014;7:123-130.

6. Miettinen M, Sobin LH, Lasota J. Gastrointestinal stromal tumors of the stomach: a clinicopathologic, immunohistochemical, and molecular genetic study of 1765 cases with long-term follow-up. Am J Surg Pathol 2005;29:52-68

7. Agaimy A. Gastrointestinal stromal tumors (GIST) from risk stratification systems to the new TNM proposal: more questions than answers? A review emphasizing the need for a standardized GIST reporting. Int $J$ Clin Exp Pathol 2010;3:461-471.

8. Kim GH, Park DY, Kim S, et al. Is it possible to differentiate gastric GISTs from gastric leiomyomas by EUS? World J Gastroenterol
2009;15:3376-3381

9. Seo SW, Hong SJ, Han JP, et al. Accuracy of a scoring system for the differential diagnosis of common gastric subepithelial tumors based on endoscopic ultrasonography. J Dig Dis 2013;14:647-653.

10. ASGE Standards of Practice Committee, Gan SI, Rajan E, et al. Role of EUS. Gastrointest Endosc 2007;66:425-434.

11. He G, Wang J, Chen B, et al. Feasibility of endoscopic submucosal dissection for upper gastrointestinal submucosal tumors treatment and value of endoscopic ultrasonography in pre-operation assess and post-operation follow-up: a prospective study of 224 cases in a single medical center. Surg Endosc 2016;30:4206-4213.

12. Gress F, Schmitt C, Savides T, et al. Interobserver agreement for EUS in the evaluation and diagnosis of submucosal masses. Gastrointest Endosc 2001:53:71-76.

13. Lee HH, Hur H, Jung H, Jeon HM, Park CH, Song KY. Analysis of 151 consecutive gastric submucosal tumors according to tumor location. J Surg Oncol 2011;104:72-75.

14. Lee JS, Kim JJ, Park SM. Laparoscopic gastric wedge resection and prophylactic antireflux surgery for a submucosal tumor of gastroesophageal junction. J Gastric Cancer 2011;11:131-134.

15. Kim GH, Ahn JY, Gong CS, et al. Efficacy of endoscopic ultrasound-guided fine-needle biopsy in gastric subepithelial tumors located in the cardia. Dig Dis Sci 2020;65:583-590.

16. Min YW, Park HN, Min BH, Choi D, Kim KM, Kim S. Preoperative predictive factors for gastrointestinal stromal tumors: analysis of 375 surgically resected gastric subepithelial tumors. J Gastrointest Surg 2015;19:631-638 